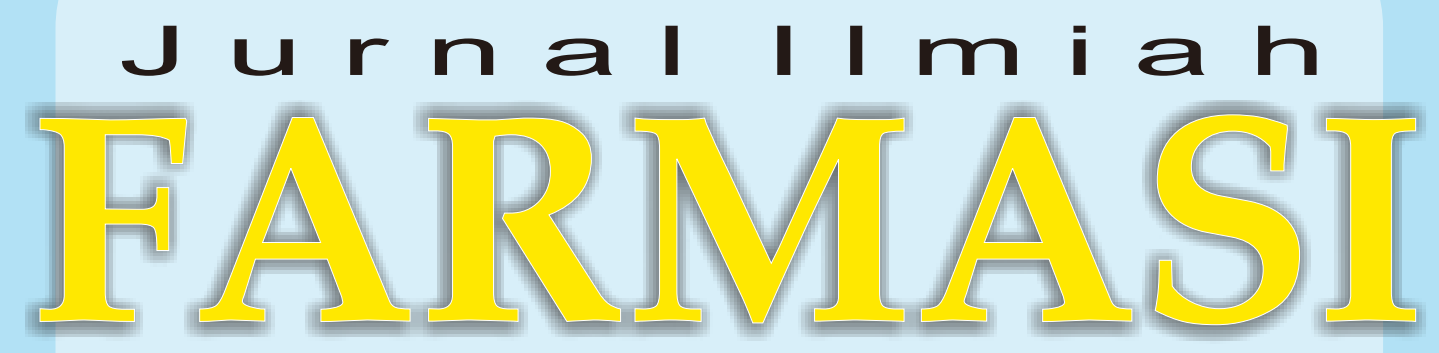

(Scientific Journal of Pharmacy) 
JURNAL ILMIAH FARMASI

(SCIENTIFIC JOURNAL OF PHARMACY)

PIMPINAN UMUM/ PENANGGUNG JAWAB

Dekan Fakultas Matematika dan Ilmu Pengetahuan Alam

Universitas Islam Indonesia

WAKIL PIMPINAN UMUM/ WAKIL PENANGGUNG JAWAB

Ketua Jurusan Farmasi FMIPA UII

\section{MITRA BESTARI}

1. Prof. Dr. Wiryatun Lestariana, Apt

2. Prof. Dr. Zullies Ikawati, Apt

3. Prof. Dr. Sudibyo Martono, Apt

4. Dr. Tedjo Yuwono, Apt

5. Prof. Dr. Dachriyanus, Apt

6. Prof. dr. Iwan Dwiprahasto, MMedSc, PhD

7. Prof. Dr. Lukman Hakim M.Sc., Apt

8. Prof. Dr. Achmad Fudholi, DEA, Apt

9. Prof. Dr. Ibnu Gholib Gandjar, DEA., Apt

\begin{tabular}{ll} 
& \multicolumn{1}{c}{ DEWAN EDITOR } \\
Ketua & : Saepudin, M.Si., Apt \\
Sekretaris & : Rochmy Istikharah, M.Sc., Apt. \\
Anggota & : Vitarani Dwi Ananda Ningrum, M.Si., Apt \\
& Okti R. Mafruhah, MSc., Apt \\
& Dimas Adhi Pradana, MSc., Apt. \\
& Fithria DA. Suryanegara, MSc., Apt. \\
& Ari Wibowo, S.Farm., Apt \\
& Arba Pramudita Ramadani, MSc., Apt. \\
& Oktavia Indrati, S.Farm., Apt.
\end{tabular}

Penerbit

Jurusan Farmasi Fakultas Matematika dan IImu Pengetahuan Alam Universitas Islam Indonesia

Alamat Penerbit Jurusan Farmasi FMIPA UII

Jl. Kaliurang Km. 14,4 Yogyakarta 55584

Telp. (0274) 896439 ext. 3047

Email: jif@uii.ac.id 


\title{
EKSPLORASI SIFAT FISIKOKIMIA 2-BENZILIDEN-SIKLOHEKSANA-1,3- DION BERDASAR PERHITUNGAN KIMIA KUANTUM SEMIEMPIRIK
}

\author{
Enade Perdana Istyastono
}

Fakultas Farmasi, Universitas Sanata Dharma, Yogyakarta

\begin{abstract}
Aromatic cyclic endione has been predicted qualitatively as anticancer agent. 2-Benzylidenecylclohexane-1,3-dione is the simplest aromatic cyclic endione compound. Physicochemistry properties of 2-benzylidene-cylclohexane-1,3-dione have been explored. The properties were explored by semiempirical quantum-chemical calculations using the computational chemistry approach. Vibrations spectrum of 2-benzylidene-cylclohexane-1,3-dione has been used to determine the best semiempirical method. The AM1 semiempirical method was found to be the most appropriate method to describe the structure of 2-benzylidene-cylclohexane-1,3-dione and explore the physicochemistry properties.
\end{abstract}

Keywords: anticancer, physicochemistry, semiempirical quantum-chemical calculation, 2benzylidene-cylclohexane-1,3-dione. 